\title{
Remote Transmission and Control Research of Visual Signal for Detecting Robot
}

\author{
SHANG ChangChun ${ }^{1,}$, , HAO Yuyu ${ }^{1}$, ZHAO Tian-peng ${ }^{1}$, ZHANG Qi $^{1}$ \\ ${ }^{1}$ Xi an University of Science and Technology, Xi an 710054, China \\ ashangchangchun@126.com
}

Keywords: Detecting Robot; Signal Robust Control; Signal Transmission; Visual System.

\begin{abstract}
Autonomous navigation is one of the key issues in the field of mobile robot. In recent years, as the robot system operation ability and the development of related technologies, such as image processing, people more and more research is not dependent on the autonomous navigation technology of GPS system, exist in applied to shade and interference such as GPS navigation system cannot be applied. In the mobile robot system, using the navigation sensor consists of laser ranging radar, and inertial measurement unit and RGB visual navigation system. In our research, a novel algorithm for visual signal long-distance transmission is proposed. The numerical analysis and simulation proves the correctness and robustness of the algorithm.
\end{abstract}

\section{Introduction}

Real-time image processing technology is popularly adopted in areas such as target tracking, machine vision, get more and more applications. The high resolution image real-time processing system, a large amount of data and algorithm complexity is high, the design of real-time processing system of image data acquisition, processing ability, external cache capacity and system performance in data transmission ability, etc. Put forward very high requirements. How to perform the huge amounts of data real-time transmission and real-time image processing system design processing is the difficulty [1-2]. Robotics is one of the most active branches of natural science research field. After decades of development, the robot in such aspects as function, configuration and performance has made rapid progress, and produced great economic benefits in the social production. At present a lot of robots are used in highly automated assembly lines, high precision high speed industrial robots is to replace human complete various simple, boring, repetitive production works. With the continuous development of science and technology and the improvement of robot technology, robot can complete the task of more and more complex. And industrial production, fixed position or movement fixed compared to the traditional industrial robots, modern robot is moving toward autonomy, mobility and flexibility, intelligent direction [3]. Their work environment has not only confined to the structured and standardized industrial environment, and more time working in the unstructured environment.

The purpose of the research on robot navigation is to make robots with independent mobility in various environments. Robot using the equipped with sensors to environmental information, build environment map, self-image, finish the tasks such as path planning. Commonly used sensors are: robot sonar, laser, infrared, visual, etc. Using sonar to form annular signal can detect obstacles around the robot, but there are larger positioning error, the laser can be conveniently surveying and mapping out the information of the scale of the environment, but the lack of detail to the environment ability and cannot be effectively closed loop detection, infrared ray can also work well in the dark, but is separate application in navigation ability insufficient. To sum up these sensors environmental information are single. Vision sensors can get very rich images (texture characteristics, color characteristics, morphological characteristics, etc.), the environmental features of ability is much stronger than the other sensors. Some of the stronger robustness algorithm can adapt to different illumination images, and remained invariant to rotation, translation, scale. The robot navigation in the natural environment is very good, can effectively solve the key problems in navigation. 
To overcome the drawbacks of high transmission rate and the transmission accuracy, we conduct research on novel robust image signal transmission algorithm in this paper. By using discrete cosine transform is proposed and the piecewise quantization sonar image data compression technology. The method dealing with the object of line is real-time imaging sonar image data. Method the implementing procedures for, first of all to discrete transform of row data, then use the energy concentration characteristic, to truncate the coefficient after the transformation, finally the truncated data are segmented quantitative processing, further improve the compression efficiency.

\section{Our Proposed Novel Algorithm}

Image Signal Compression. Proposed DTC transformation and piecewise quantization based real-time processing system of sonar image data compression method mainly includes two parts: in part line sonar imaging processing subsystem of image data compression and decompression part in sonar display control subsystem. The formulas $1 \sim 2$ show the transformation.

$$
\begin{aligned}
& y(k)=w(k) \sum_{n=1}^{N} x(n) \cos \left(\frac{\pi(2 n-1)(k-1)}{2 N}\right), k=1,2, \ldots, N \\
& x(n)=\sum_{k=1}^{N} w(k) \mathrm{y}(k) \cos \left(\frac{\pi(2 n-1)(k-1)}{2 N}\right), \mathrm{n}=1,2, \ldots, N
\end{aligned}
$$

Because of the key components of parallel application software commonly used have integrated in parallel vector library, so can be concentrated in all kinds of testing, validation and optimization, the existing technology to ensure its quality and operation effect, thus improve the quality and efficacy of parallel applications. And the lower level of object is mainly used as above the level of support environment. Abstract data layer contains the applications of parallel data type, it is simply a set of parallel data with classified information is encapsulated objects and its operator [4-5]. It passes its information and operation data partition and parallel support layer data classification, data communication and data operator. DTC transformation is often used in signal processing and image processing, especially used for signal or image lossy data compression. Its characteristic is the most natural signal after transform its energy mainly concentrated in the low frequency part, but seldom from the high frequency part of the energy. We have carried on the processing to the actual sound figure, a row of image data for analysis. First of all, defines the strength of the DCT coefficients:

$$
I(k)=20 \lg ([a b s(y(k))] /[\max (a b s(y(k)))])
$$

Restore lines image signal and the original image signal difference evaluation methods mainly include two categories, subjective evaluation and objective evaluation. Subjective evaluation is made up of a number of different observers for subjective evaluation, the evaluation for statistical analysis.

$$
M S E=\frac{1}{N} \sum_{n=1}^{N}[g(n)-f(n)]^{2}
$$

Segmenting the truncated data is to do quantitative processing and further compression. Is the main basis, even if in the paragraph of interception, its high energy distribution is still the low frequency part and high frequency part is low and therefore in low frequency paragraph for quantitative processing of high precision, high frequency precision low quantitative processing paragraphs. This can be further compressed. The regularization process is shown below:

$$
C C=\left(\sum_{n=1}^{N}[f(n) \cdot g(n)]\right) / \sum_{n=1}^{N} f(n)^{2}
$$

Visual Navigation and Control. In general, it is hard to image sequence based on monocular camera to restore the depth of the object information, causing fuzzy characteristics of the scale of the scene depth, it is also a monocular vision system to realize the main difficulties faced six degrees of freedom navigation [6-7]. Intuitive sense, in order to solve the problem of camera movement scale fuzzy, need to get the scene geometry characteristic of information or the camera movement velocity 
amplitude information. Until recently, the line of sight of repeated observations difference is used to estimate the depth of the scene characteristic information, called the parallax method. As is known to all, if the scene of a static feature points last, repeated observation, so as the change of body position, observed the pixel coordinates of the feature points will change. If the camera parameters are known based on the change of feature point image coordinates, to restore the feature point position in world coordinates, it is also the basic principle of parallax method. The following figure describes this.

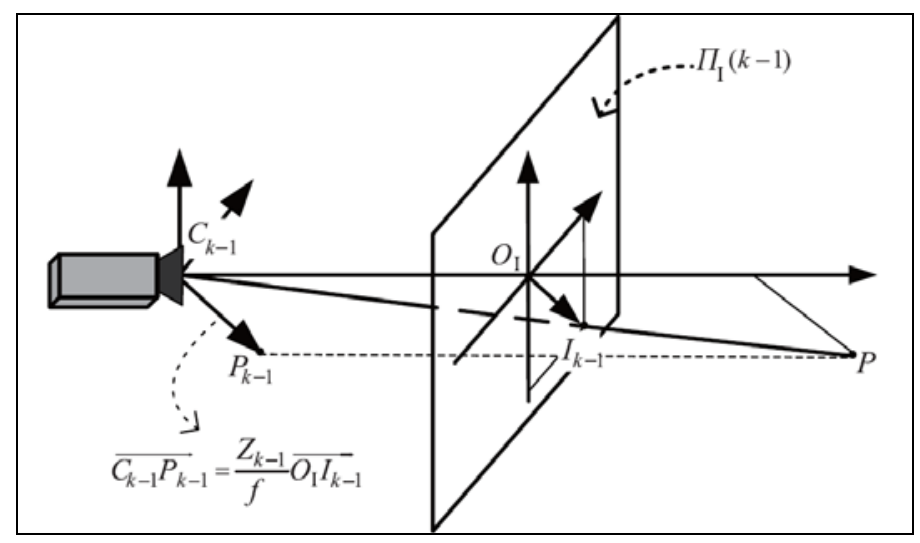

Fig. 1The Flowchart of the Visual System

Commonly used parametric model is Euclidean XYZ coordinate parameterized model, the model of feature points directly to the world coordinate system coordinates augmented to state variables, so as to realize the scene feature location estimation. When the scene feature optical center far distance from the camera or parallax angle is small, the model will introduce greater error shown in the Fig 2.

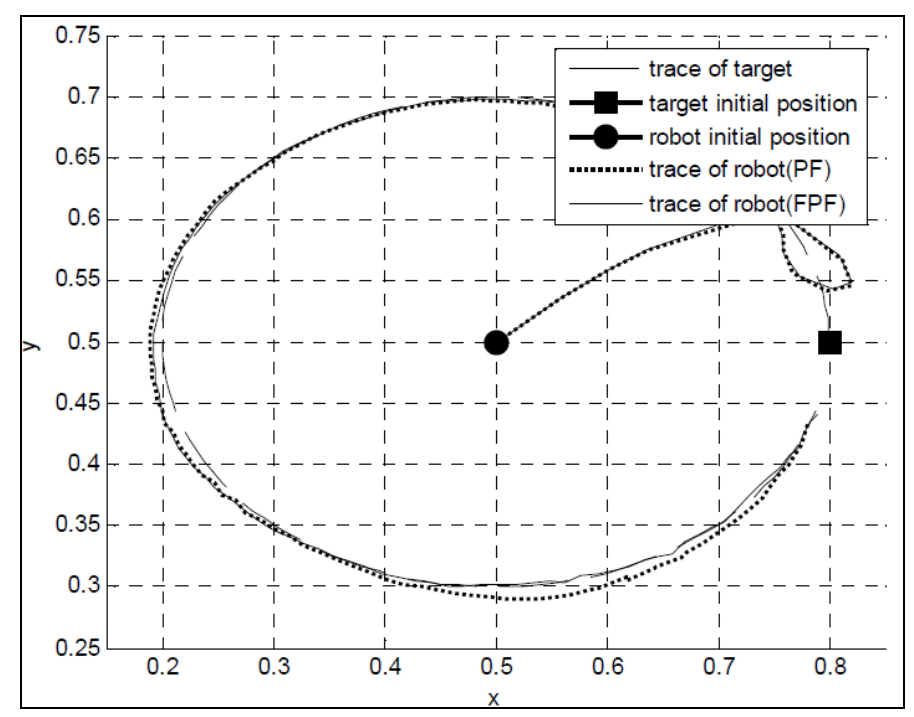

Fig. 2The Illustration of Visual Tracking Figure

First based on monocular RGB image sequence, we finish the establishment of the depth of the visual scene feature points incremental parameterized model, by reducing state variable dimension, improve the system observability, and thus improve the performance of the monocular vision navigation. Based on this, we will get the depth of the data access as observed in the depth of the incremental model, in order to realize the motion estimation filter the closed-loop structure design, thereby enhancing model for the robustness of the disturbance of environment. Monocular camera pose estimation problem is according to the camera in the image sequence of discrete sampling point to be treated, through the establishment of the image feature matching and camera movement between the constraint relationships between, calculate the camera motion parameters. Similarly, based on the Euclidean observability analysis of parametric model, can find their corresponding linear model of Taylor expansion is significant sex cannot be guaranteed. Get position, therefore, difficult to guarantee the global optimal solution of parameters. By increasing the relation of constraint on camera movement, can narrow the scope of the optimization search, so as to improve 
the accuracy of posture calculating. Due to the inherent characteristics of the pinhole camera model, using the polar constraint solving camera speed, scale fuzzy uncertainty, therefore, pose estimation based on image sequence feature points matching algorithm is a kind of incomplete pose estimation method. The experiment of the method is shown in the next chapter.

\section{Experiment and Result}

Experiment simulation and real environment is divided into two parts. Panoramic vision is validated by simulation experiments. The characteristics of big vision in problem solving, for ordinary visual advantages are in real environment experiments this article proposed the method based on improved on the engineering feasibility and validity. The figure 3 shows the result.
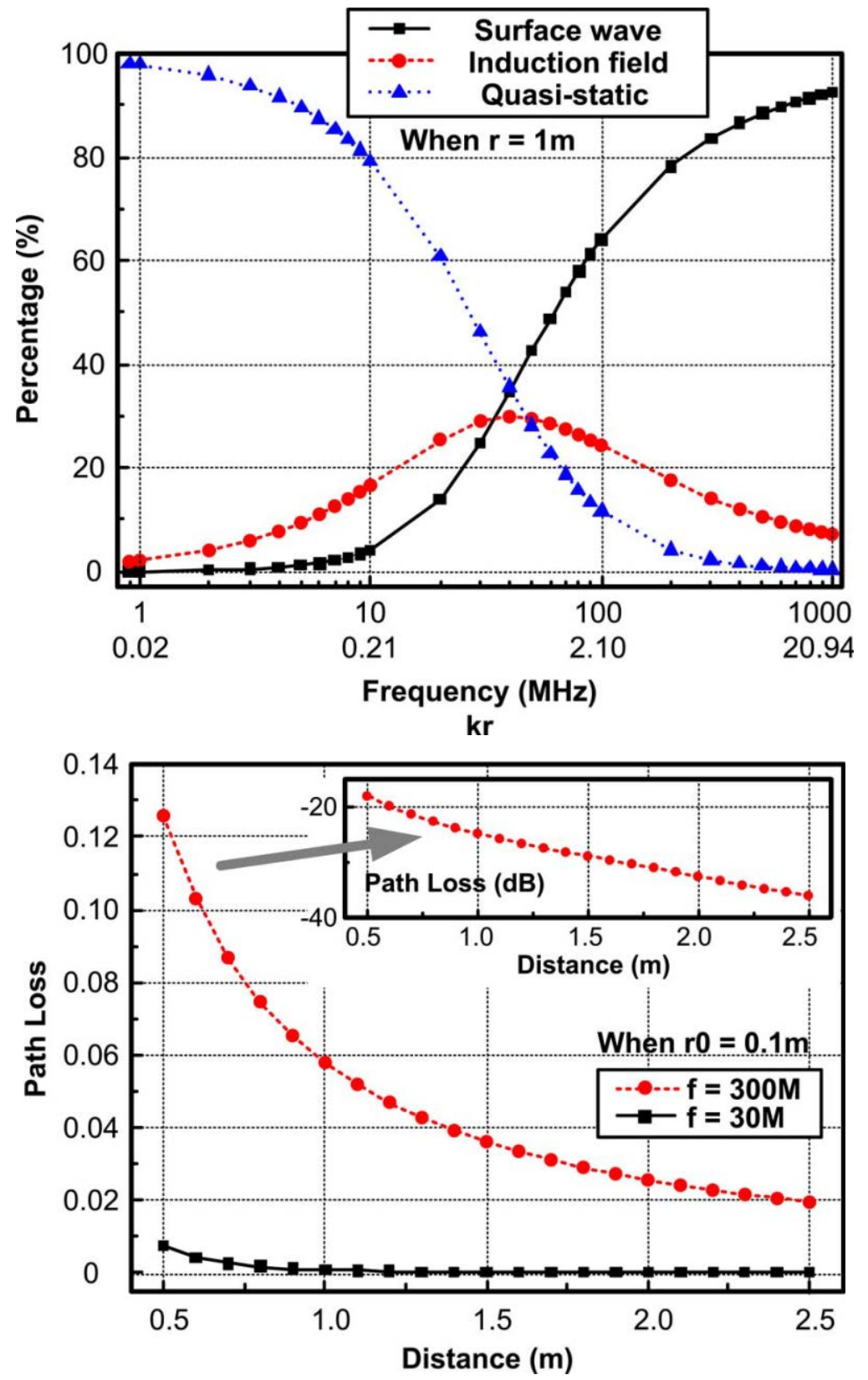

Fig. 3The Result of the Experiment

\section{Conclusion and Summary}

Conventional monocular camera pose estimation algorithm exists displacement scale factor of uncertainty, so need to create a parameterized model of feature points, general Euclidean parametric model and inverse depth with high dimension, observability is no guarantee that the defects, this 
paper proposed the incremental parametric model based on parameterized, the depth of the feature point information can reduce the state dimension as much as possible at the same time, ensure consistent observability of the system state, so as to realize the monocular camera 6-d pose estimation results of convergence. In real-time processing imaging sonar systems, some need to do image data wireless transmission platform, the typical such as semi-submersible craft, imaging subsystem on the aircraft do real-time imaging, and use the radio channel to transmit the data to the mother ship for real-time display. Limited channel bandwidth and need to do image data compression and decompression processing. Our proposed method solve the issue properly, we plan to conduct more related work in the near future to optimize the current status of the approach.

\section{References}

[1] Chiappetta M J, Frankel J P. Celestial navigation system for an autonomous robot: U.S. Patent 7,706,917[P]. 2010-4-27.

[2] Kurz, Daniel, et al. "An outdoor ground truth evaluation dataset for sensor-aided visual handheld camera localization." Mixed and Augmented Reality (ISMAR), 2013 IEEE International Symposium on. IEEE, 2013.

[3] Raty T D. Survey on contemporary remote surveillance systems for public safety[J]. Systems, Man, and Cybernetics, Part C: Applications and Reviews, IEEE Transactions on, 2010, 40(5): 493-515.

[4] Wang, Haoxiang, Ferdinand Shkjezi, and Ela Hoxha. "Distance metric learning for multi-camera people matching." In Advanced Computational Intelligence (ICACI), 2013 Sixth International Conference on, pp. 140-143. IEEE, 2013.

[5] Ren, X., et al. "A New lunar global topographic map products from Chang'E-2 Stereo Camera Image Data." European Planetary Science Congress 2014, EPSC Abstracts, Vol. 9, id. EPSC2014-344. Vol. 9. 2014.

[6] McMahon, Andrew Kenneth John. "Systems and methods for transmitting and receiving array camera image data." U.S. Patent No. 8,692,893. 8 Apr. 2014.

[7] Noda, Masafumi, et al. "Vehicle ego-localization by matching in-vehicle camera images to an aerial image." Computer Vision-ACCV 2010 Workshops. Springer Berlin Heidelberg, 2011. 